\title{
Interaction models between humans and lower-limbs exoskeletons applied to robotic neurorehabilitation
}

\author{
Denis Mosconi ${ }^{1}$, Adriano A. G. Siqueira ${ }^{2}$ \\ ${ }^{1}$ Mechanical Engineering Department - University of São Paulo (USP) \\ São Carlos - SP - Brazil \\ ${ }^{2}$ Center of Engineering Applied to Healthy, São Carlos School of Engineering, \\ University of São Paulo (USP), São Carlos - SP - Brazil \\ denis.mosconieifsp.edu.br, siqueiraesc.usp.br
}

\begin{abstract}
Development of interaction controls applied to robots of roboticassisted therapy is a challenge, as it requires physical contact between human and robots, in addition to demanding a high cost of time and resources. The purpose of this work was to develop a human-exoskeleton interaction model and a simulation algorithm for development, validation and testing of interaction controls applied in robotic rehabilitation of lower-limbs. Two simulations with different interaction models and controls were run, combining experimental and computational data. The results obtained proved that both the interaction model and the simulation algorithm are feasible, useful, improving flexibility and agility for developing of interaction controls.
\end{abstract}

\section{Student level: MSc}

Date of conclusion: $2020-03-23$

\section{Introduction}

Stroke is a noncommunicable disease of the cardiovascular type, caused by the suspension of the blood supply to the brain because a bleeding (hemorrhagic stroke) or a clot (ischemic stroke). In the last 15 years, the disease was the second leading cause of death in the world. Annually, about 15 million people worldwide suffer a stroke (every two seconds someone in the world has a stroke). Of these, 5 million die and another 5 million remain permanently disabled [Mackay and Mensah 2004, WHO 2011, WHO 2018].

According to the Brazilian Ministry of Health, $10 \%$ of the deaths in the country are caused by stroke, and in 2016 there were 188,223 hospitalizations and 40,019 deaths caused by the disease [MS 2018]. According to [SAFANELLI et al. 2019], the mean cost per patient with any type of stroke is approximately US\$ 4,770.00 in a public hospital in Brazil.

The World Health Organization forecasts a continuous increase in stroke occurrences. Some factors help explain this upward trend: sedentary lifestyle, incorrect diet, alcohol, tobacco and population aging. It is expected that by 2030 there will be approximately 200 million people around the world with some motor disability due to stroke, which is approximately the number of the current Brazilian population [WHO 2011].

The severity and localization of the stroke in the brain determines the type and severity of the disability. Some examples of disabilities are the difficulty of speaking and understanding, vision problems and weakness in arms and legs, increasing the 
patient dependency on other people to execute activities of daily living (ADL) such as walking, dressing, writing and eating. The victim not only has their motor abilities affected by the stroke, but also their psychological ones, which can result in depression and demotivation. Moreover, in underdeveloped countries, families whose a member becomes a stroke victim are at serious risk of falling into extreme poverty [Rangel et al. 2013, Ramos-Lima et al. 2018].

Then, in order to recover the motor capabilities of a stroke survivor (e.g. gait pattern, equilibrium) and allow victims to return to their normal lives, physical therapy involving rehabilitation makes necessary. Such therapy of rehabilitation consists in the execution of specific movements in order to provoke motor neuroplasticity to the patient, improving motor recovery and minimizing functional deficits [Diaz et al. 2011].

However, the growing demand requires for new methods capable of increasing the effectiveness and reach of these treatments. In this context, robotic-assisted therapy is emerging as a proponent area to development of rehabilitation activities. Robotic rehabilitation can replace the physical effort of the therapist, ensuring more intensive and repetitive movements, with uniformity, for long-time routines, reliable storage of data and eliminating the need for several therapists per patient, especially in gait rehabilitation, increasing the manpower availability. In addition, rehabilitation robots are able to meet the Assist-as-Needed Paradigm, that is to assist the patient to perform the movement in therapy only when necessary, assuming a transparent behavior when the subject can perform the task on its own, contributing to cortical reorganization through neuroplasticity [Diaz et al. 2011, Jutinico et al. 2017, Androwis et al. 2018].

The interaction between humans and robots is a challenge in the field of robotic rehabilitation, since such interaction should ensure the safety of the patient, meet the therapy requirements for each specific subject and satisfy the assist-as-needed paradigm, predicting the intention of movement of the user. Several interaction controls have been studied in order to reduce the risks and ensure the treatment efficacy: EMG-driven adaptive impedance control [Peña 2017], control strategy based on kinetic motor primitives [Nunes et al. 2018], performance-based adaptive assistance controller [Bayon et al. 2018], motor intention decoding algorithm [Pastore et al. 2018], Markovian robust compliance control [Jutinico et al. 2018], impedance control using functional electrical stimulation [Kim and Kim 2018].

But the validation and testing of these controllers is still a challenge, as the tests involved demand physical contact between a user and a robot, which can put them at risk of an accident, in addition to demanding time and resources in the preparation of such tests. Then, how to do this with reduced cost, low time consumption and without putting patients at risk? A possible solution is the use of interaction models: Computational models of the human-robot set, attending both the patient characteristics and the rehabilitation robot, and able to provide reliable data on the interaction controls developed. Such interaction models allow the interaction controls used in robotic rehabilitation to be developed, adjusted and tested, minimizing the need for physical tests involving a user and a robot, which reduces the risk of accidents in addition to saving design time.

The purpose of this work was to develop a patient-exoskeleton interaction model that reproduces the dynamics of a subject wearing a lower limb exoskeleton and a forward 
dynamics-based simulation algorithm that can be used for development, validation and testing of interaction controls applied in robotic rehabilitation of lower-limbs.

This paper is organized as follows. In Section 2 some related works are shown and discussed. In Section 3 the development of the interaction model and the simulation algorithm is presented. In Section 4 some simulation varying the interaction model and the interaction control are performed. Lastly, conclusions are shown in Section 5.

\section{State of Art}

In this section we present some related work that recurred to computational models and simulation algorithms to perform biomechanical analysis and human-robot interaction control development for rehabilitation purposes.

[Delp et al. 2007, Seth et al. 2018] developed the OpenSim ${ }^{1}$ : a freely available, open-source software to modeling, simulating and analyzing the neuromusculoskeletal system and its movements. Despite dealing very well with the purpose for which it was developed, the software by itself does not allow the development of interaction controls in an agile and flexible way. Another such software is the AnyBody Modeling System ${ }^{2}$ however it is closed and proprietary [Damsgaard et al. 2006]. A comparison study between OpenSim and AnyBody was carried out by [Trinler et al. 2019]. A C++, Python and MATLAB library to analyze and simulate human body biomechanics was developed by [Michaud and Begon 2021], it allows simulations based on the inverse or direct flow [Kainz et al. 2016] but does not have a Graphical User Interface.

[de Sousa et al. 2019] combined the skills of OpenSim and MATLAB to create a simulation environment to reproduce the feedback PID controls of torque and FES applied to a hybrid neuroprosthesis, in this case the human control was not considered. [Mansouri and Reinbolt 2012] developed a platform for dynamic simulation and control of the human movement based on OpenSim and Simulink, allowing simulations with both open and closed muscles controls, not taking into account the use of robots. A MATLABOpenSim based simulation framework for virtual prototyping of robotic exoskeleton, with focus in parameter optimization, was developed by [Agarwal et al. 2016].

Biomechanical computational models and simulations environments were also used to estimate the torques and the states of humans wearing assistive devices (exoskeleton, prosthesis or orthosis) enabling the development of interaction controls based on impedance [Peña et al. 2019], kinetic motor primitives [NUNES et al. 2017], [Nunes et al. 2018], backstepping sliding mode [Khamar and Edrisi 2018], robustness [Guzmán et al. 2017], PID feedback [Handford and Srinivasan 2018] and FES cycling [de Sousa et al. 2016].

The cited works proved that the use of computational models and simulation algorithms is useful. However, such works deal with specifics models and simulators that serve only to the aim of the work in question, not showing flexibility and comprehensiveness, and therefore incapable of being used by other rehabilitation engineers to simulate different models and controls.

\footnotetext{
${ }^{1}$ http://opensim.stanford.edu

${ }^{2}$ https://www.anybodytech.com
} 


\section{Methodology}

In this section we discuss the development of the interaction model and simulation algorithm. The OpenSim version 3.3 and the MATLAB R2017b were the platforms where the developments and simulations took place.

\subsection{Human-exoskeleton Interaction Model}

The human-exoskeleton interaction model consists of a computational neuromusculoskeletal model (NMS) that represents the human lower limb biomechanics, in whose hip and knee joints coordinate actuators were coupled in order to reproduce computationally the functioning of an exoskeleton.

In this work, two NMS models provided by OpenSim were used: the leg6dof9musc (Figure 1b) that is a three-dimensional, 6 degree-of-freedom model with 9 musculotendon actuators and represents a single leg, pelvis, femur, tibia and foot and the gait2392 (Figure 1c) that is a three-dimensional, 23 degree-of-freedom model that has 92 musculotendon actuators to represent 76 muscles in the lower extremities and torso, the bones are modeled as rigid bodies [Delp et al. 1990]. The default version of this model represents a subject with mass of $75.16 \mathrm{~kg}$ and a height of $1.8 \mathrm{~m}$. In the NMS depicted in the Figure $1 \mathrm{a}$ and $\mathrm{b}$, the red lines represent the muscles, the blue discs represent the robot actuator and the green arrow indicates the sagittal direction of the movements.

The virtual model of the exoskeleton (represented by the coordinate actuators) was developed based on the ExoTAO (Figure 1a), a lower limbs exoskeleton introduced by [dos Santos et al. 2017b] that consists of a lightweight tubular structure, with six independent and free joints which promotes movements in the sagittal plane. Each joint is equipped with an absolute encoder AksIM ${ }^{\mathrm{TM}}$ (from Renishaw), except for the knee joint where the series elastic actuator (SEA) designed by [dos Santos et al. 2017a] is used. In this work, some simplifications were made: the actuators are ideal (no mass, delay or losses), the axes of the joints of the robot and the user are collinear, the torque is applied directly to the human joint in question.

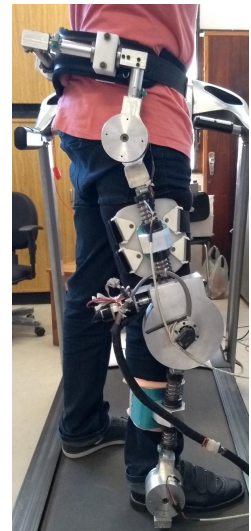

(a) ExoTAO.

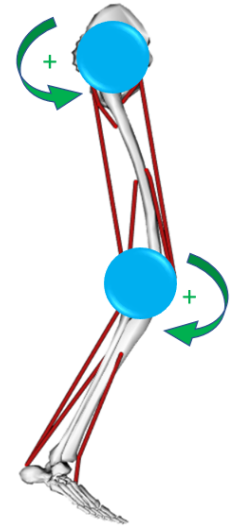

(b)

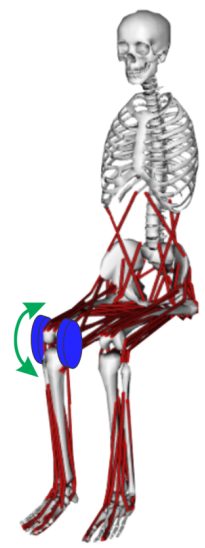

(c) Gait2392

Leg6dof9musc

Figure 1. (a) A subject wearing the full configuration of ExOTAO. (b) and (c) interaction models. 
The anthropometry of the interaction models are fitted to a particular subject using the Scale Tool $^{3}$ from OpenSim.

\subsection{Forward Dynamics-based Simulation Algorithm}

In order to reproduce the interaction controls, an algorithm based in forward dynamics was developed, combining the capabilities of modeling and simulation from OpenSim with those of control design from MATLAB. Figure 2 presents a flowchart of the algorithm.

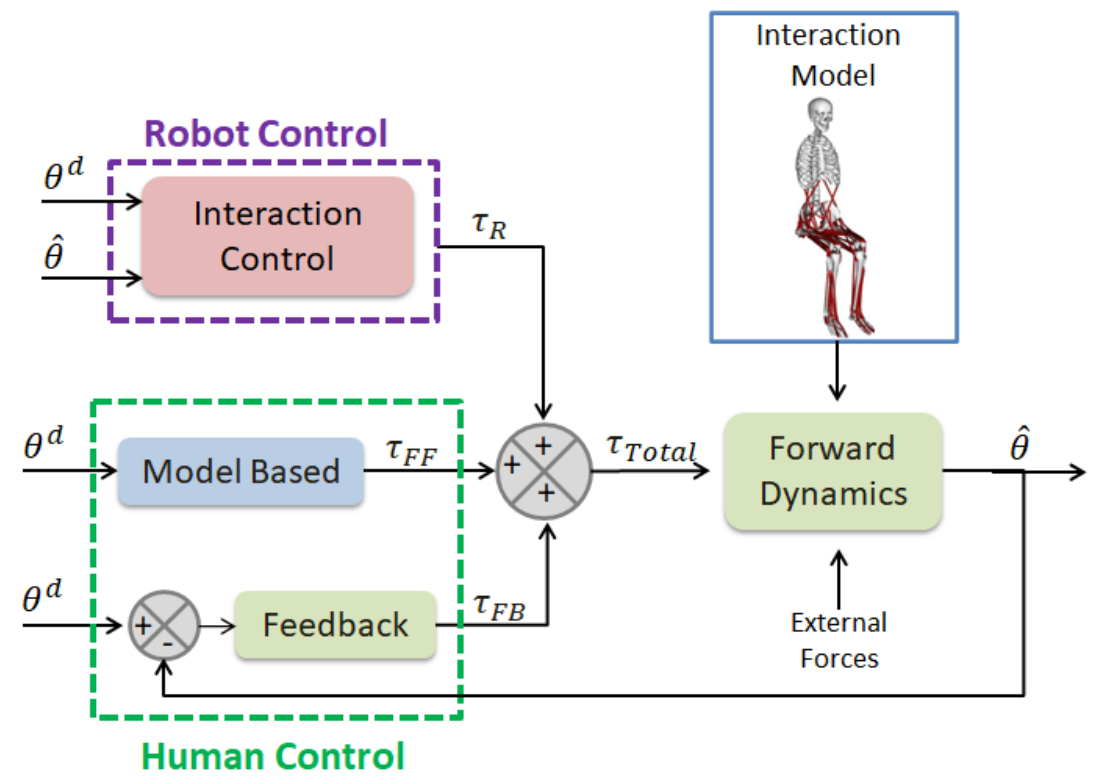

Figure 2. Forward dynamics-based algorithm to simulation of the interaction controls applied to the human-exoskeleton interaction model.

The Robot Control block contains the interaction control (e.g. impedance control, fuzzy control, primitive-based control) that governs the actions of the exoskeleton. Its inputs are the desired position of the joints $\left(\theta^{d}\right)$ and the simulated position of the joints $(\hat{\theta})$. The output are the torques of the robot actuators $\left(\tau_{R}\right)$.

The Human Control simulates the human behavior, and is based on the feedforward-feedback control and internal model concept [Frey et al. 2011, Wolpert et al. 1998]. Such a concept states that the human being has in the cerebellum an inverted dynamics model of the human body, so that, given the desired motor action (e.g. walking), and the current state of the limbs, the inverse internal model computes the excitation to be sent to the muscles, which is represented in the Figure 2 as a feedforward loop that provides the forward torque $\left(\tau_{F F}\right)$. If the performance of the predicted movement is affected by disturbances, causing a trajectory error, which is perceived by the individual through afferent signals, a feedback control loop provides a correction torque $\left(\tau_{F B}\right)$ in order to ensure the desired movement be accomplished. In this work, the feedforward loop consists of the Inverse Dynamics Tool from OpenSim and the feedback loop is a conventional proportional-integral-derivative (PID) control whose gains were determined by trial-and-error.

\footnotetext{
${ }^{3}$ https://simtk-confluence.stanford.edu/display/OpenSim/Scaling
} 
The External Forces, as its name says, are forces that do not come from the human-robot system, but come from another sources like as the ground (ground reaction forces), additional loads placed by the therapist, and contact forces with the environment.

The torques from robot and the ones from the user are summed resulting in a torque total $\left(\tau_{\text {Total }}\right)$ that is applied to the coordinate actuators of the interaction model. Then, the Forward Dynamics routine determines the real position of the joints $(\hat{\theta})$, realizing the numerical integration of the dynamics equation of the model (Equation 1), considering the inputs of torque total and the external forces. The integration routine used in this work was ODE45 from MATLAB.

$$
\ddot{q}=M(q)^{-1}\left(\tau_{\text {Total }}-V(q, \dot{q})-F(\dot{q})-G(q)\right)
$$

Where $M(q)$ is the inertia matrix, $V(q, \dot{q})$ is the Coriolis/centripetal vector, $F(\dot{q})$ is the friction vector, $G(q)$ is the gravitational forces vector, $\tau_{\text {Total }}$ are the applied forces and $\ddot{q}$ is the joint accelerations.

\subsection{Simulations}

In this work we carried out simulations with the two interaction models presented. With gait2392-based we simulated a rehabilitation movement of knee extension-flexion and with leg6dof9musc-based we simulated the swing of gait.

The simulations were performed on a computer with Intel®Core ${ }^{\mathrm{TM}} \mathrm{i} 7-10510 \mathrm{U}$ 2.30 GHz processor, 8.00 GB of RAM, 2.00 GB dedicated video card, 512 GB SSD PCIe 3.0 x2 NVMe (M.2 2280) and Windows 10 Pro 64 bits.

\section{Results and Discussion}

In the first simulation, using the gait2392-based model, an experimental procedure was conducted with a healthy subject wearing the knee orthosis from ExoTAO and performing sinusoidal movements of flexion and extension of the right knee. The orthosis was controlled by and impedance control (Equation 2) and used in two different modes: assistive and resistive. In the assistive mode, the orthosis helped the subject to perform the desired movement and in the resistive mode the orthosis applied a torque in order to oppose the movement performed by the user, presenting itself as a resistance.

$$
\tau_{R}=K_{R}\left(\theta^{d}-\hat{\theta}\right)-B_{R} \dot{\theta}
$$

Where $\tau_{R}, K_{R}$ and $B_{R}$ are the torque, virtual stiffness virtual damping coefficient of the orthosis, respectively and $\dot{\theta}$ is the angular velocity of the joint.

The knee angular position and the orthosis torque were measured during the experimental procedure. The angular position was used as a reference ( $\theta^{d}$ in Figure 2$)$, so we expect the interaction model to perform the same movement performed by the subject during the experiment $\left(\hat{\theta}=\theta^{d}\right)$. In this case, the Robot Control block (see Figure 2) does not contain an explicit control law, but the vector of the orthosis torques, measured during the experiment. As there is not contact with the ground or the environment, the external forces were considered null in this case. 
Analyzing Figure $3 \mathrm{a}$ it is possible to notice that the model performed the same movement as the subject, both for the assistive and resistive modes. The absolute tracking error is less than on degree (Figure 3b), with root mean square (RMS) errors equal to 0.39 and 0.37 degrees for the assistive and resistive modes, respectively.

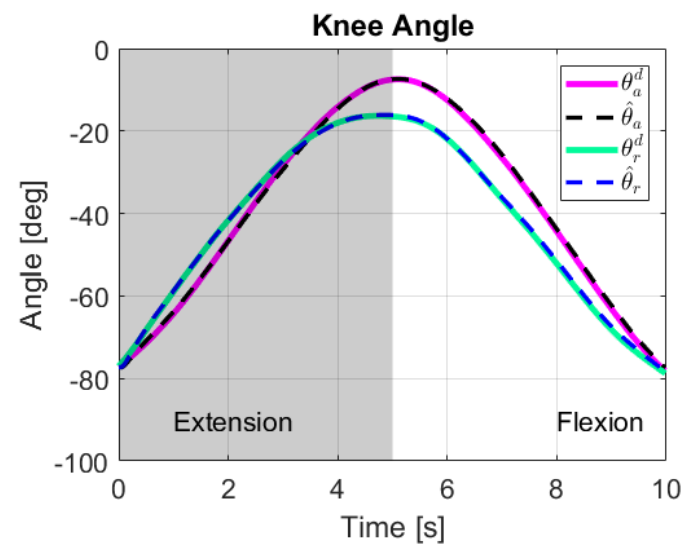

(a) Knee angle

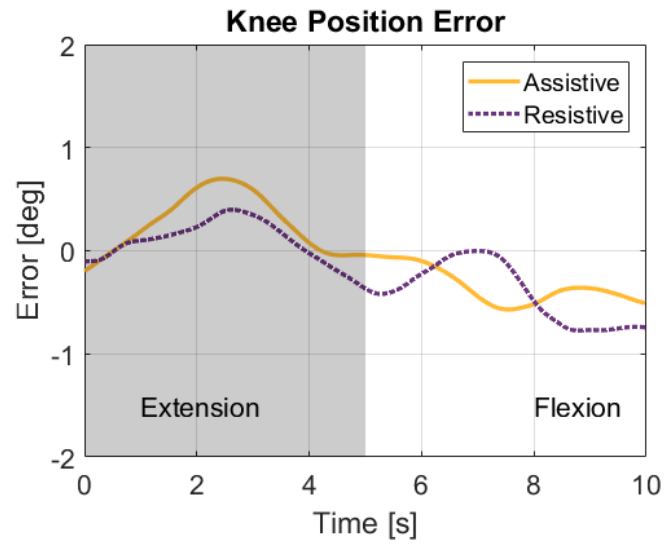

(b) Knee position error

Figure 3. (a) $\theta_{a}^{d}$ and $\theta_{r}^{d}$ are the references for the assistive and resistive mode, respectively and $\hat{\theta}_{a}$ and $\hat{\theta}_{d}$ are the movement performed by the model for the assistive and resistive mode, respectively (b) presents the tracking error for each mode.

Observing Figure $4 \mathrm{a}$ it can be seen that the orthosis applied a low torque $\left(\tau_{R}\right)$ what is expected, since the subject is healthy. A low feedback torque $\left(\tau_{F B}\right)$ was also applied by the user, what permits to conclude that the feedforward torque $\left(\tau_{F F}\right)$ was well estimated. In Figure $4 \mathrm{~b}$ the feedforward torque has been increased, because in addition to executing the movement, the user must overcome the robot opposition force, evidenced by $\tau_{R}$ that has a direction opposite to $\tau_{F F}$. In this case, the feedback torque was also increased in order to correct tracking errors and ensure that the model followed the desired trajectory well.

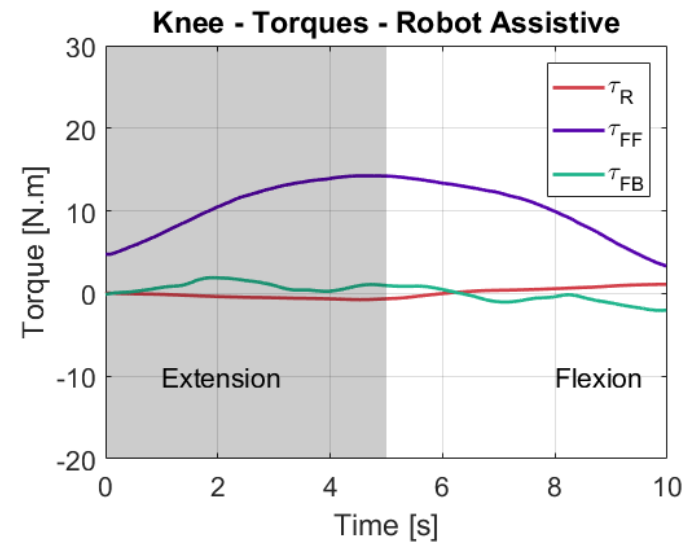

(a) Knee torques assistive

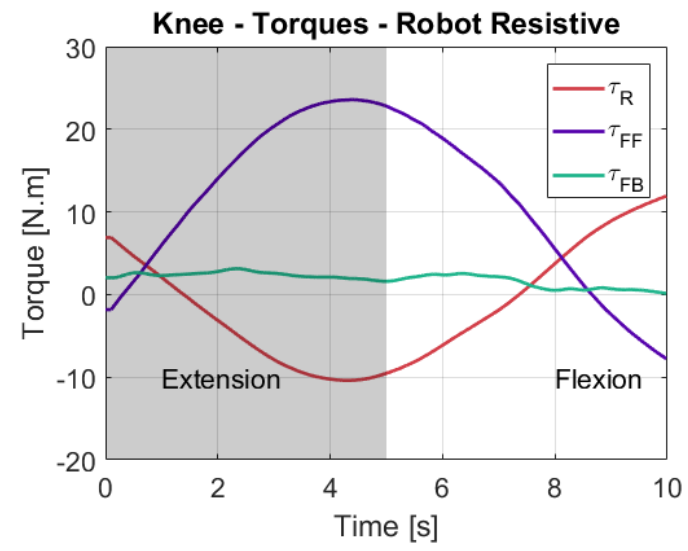

(b) Knee torques resistive

Figure 4. Knee Torques for (a) assistive and (b) resistive modes.

In the second simulation, using the leg6dof9musc-based model, an experimental 
procedure with a healthy subject wearing the ExoTAO and walking on a treadmill at a speed of $2 \mathrm{~km} / \mathrm{h}$ for 3 minutes was conducted. As in the previous experiment, the exoskeleton torques and joint positions were measured, but for this simulation, only the measured positions were used and, as in the previous simulation, taken as a reference $\left(\theta^{d}\right.$ in Figure 2).

The Robot Control block contains an impedance control (Equation 3) law that determines the torques of the hip and knee actuators of the exoskeleton computational model.

$$
\left[\begin{array}{c}
\tau_{H} \\
\tau_{K}
\end{array}\right]=\left[\begin{array}{cc}
K_{H} & 0 \\
0 & K_{K}
\end{array}\right]\left[\begin{array}{l}
e_{H} \\
e_{K}
\end{array}\right]-\left[\begin{array}{cc}
B_{H} & 0 \\
0 & B_{K}
\end{array}\right]\left[\begin{array}{c}
\dot{\theta}_{H} \\
\dot{\theta}_{K}
\end{array}\right]
$$

In the variables of the equation above, the subscripts $H$ and $K$ refer to hip and knee, respectively. $\tau_{i}$ is the torque provided by the exoskeleton (where $i \in\{H, K\}$ ). $K_{i}$ is the virtual stiffness and $B_{i}$ is de virtual damping coefficient of the robot. $\dot{\theta}_{i}$ is the angular velocity of the joint and $e_{i}$ is the position error.

The model was simulated as the user having some weakness. For this, the total human torque decreased in $20 \%$. As no ground reaction forces measurement were made, only the swing phase of the right leg was considered during the gait movement.

Analyzing (Figure 5a) it is possible to notice that the reference was well tracked both for the hip and knee joints. The absolute tracking error is less than 1.5 degree (Figure $5 \mathrm{~b}$ ), with RMS errors equal to 0.38 and 0.75 degrees for the hip and knee joints, respectively.

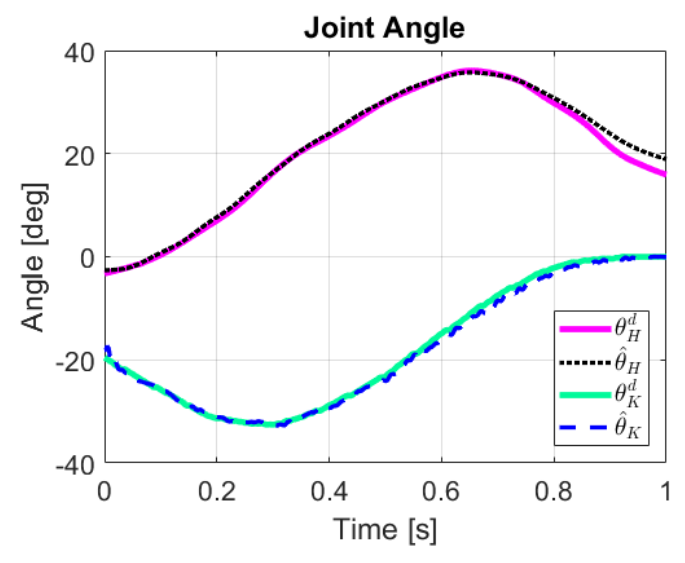

(a) Joint angle

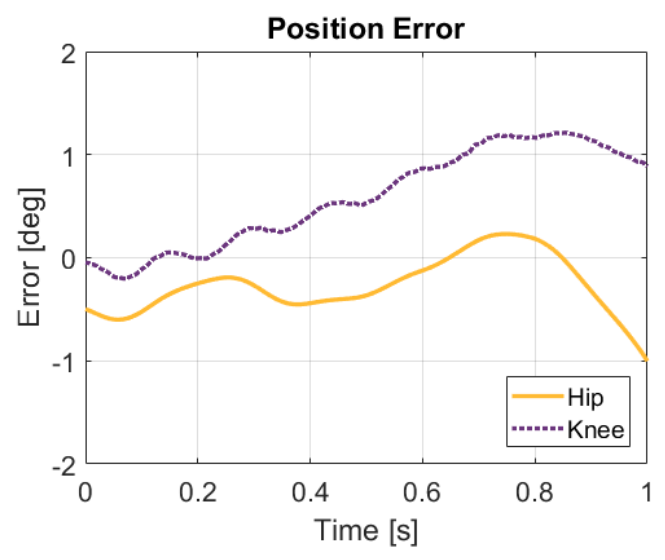

(b) Position error

Figure 5. (a) $\theta_{H}^{d}$ and $\theta_{K}^{d}$ are the references for the hip and knee joints, respectively and $\hat{\theta}_{H}$ and $\hat{\theta}_{K}$ are the movement performed by the model related to the hip and knee joints, respectively (b) presents the tracking error for each joint.

Observing Figure 6 is possible verify that the impedance control provided a complementary torque that helped the user to accomplish the movement with a minor deviation from the reference. The torques obtained from the impedance control are similar in shape to those obtained through the PID control, which is also an error-based control, proving the consistency of both controls. The torque applied to the hip is greater in 
module than that applied to the knee, since the mass and moment of inertia of the bodies connected to the hip joint are greater than the ones of the bodies connected to the knee joint.

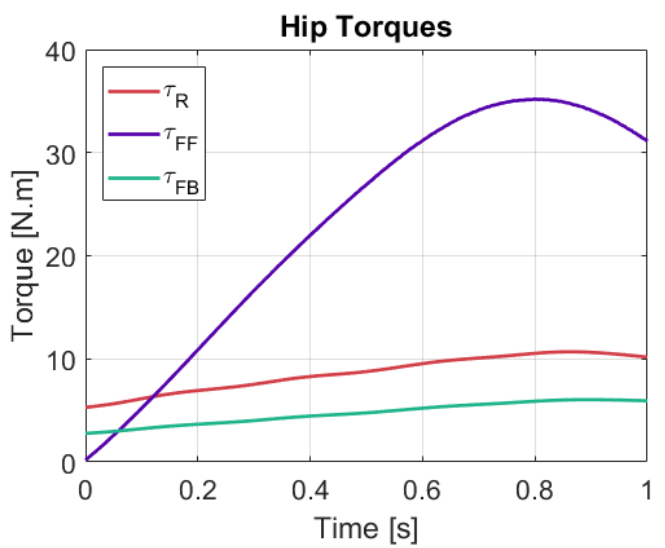

(a) Hip torques

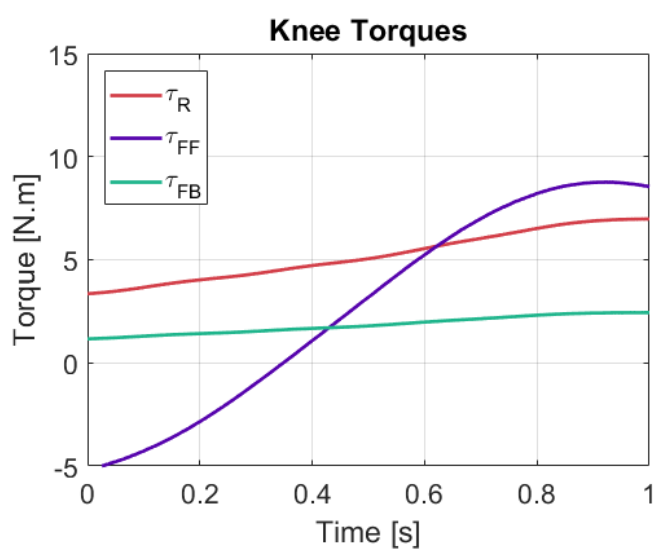

(b) Knee torques

Figure 6. Torques for (a) hip and (b) knee joints.

The first simulation presents in this work took about 2.5 minutes to be accomplished while the second simulation took about 7 minutes, evidencing the agility of the algorithm whose consistency is proved by coherency of the results obtained. Finally, the ability to deal with more than one type of interaction model, experimental and computational data, explicit or not explicit controls, as well as robot and human control, proves the flexibility of the patient-exoskeleton interaction model and simulation algorithm proposed.

\section{Conclusion}

In this work we proposed a patient-exoskeleton interaction model and a simulation algorithm able to be used for development, validation and testing of interaction controls applied in robotic rehabilitation of lower-limbs.

The results obtained with the application of these resources proved that they are feasible and useful for the purposes they should serve. The short time of the simulations evidence they agility and the fact that it handles more than one model, interaction control, experimental and computational data evidence its flexibility. The coherence of the results obtained proves its effectiveness.

For future work, it is suggested that a model optimization routine be included, in order to reduce the degree of uncertainty of the model in relation to a specific subject.

\section{Acknowledgments}

This work is supported by Pro-Rectory of Research of University of São Paulo, Coordenação de Aperfeiçoamento de Pessoal de Nível Superior - Brasil (CAPES) - Finance Code 001, PGPTA, under grant 3457/2014, and São Paulo Research Foundation (FAPESP) under grant 2013/14756-0 and 2019/05937-7. 


\section{References}

Agarwal, P., Neptune, R. R., and Deshpande, A. D. (2016). A simulation framework for virtual prototyping of robotic exoskeletons. Journal of Biomechanical Engineering, 138(6):1-15.

Androwis, G. J., Pikar, R., Ramanujam, A., and Nolan, K. J. (2018). Electromyography assessment during gait in a robotic exoskeleton for acute stroke. Frontiers in Neurology, 9:630.

Bayon, C., Fricke, S. S., Rocon, E., van der Kooij, H., and van Asseldonk, E. H. F. (2018). Performance-based adaptive assistance for diverse subtasks of walking in a robotic gait trainer: Description of a new controller and preliminary results. In 2018 7th IEEE International Conference on Biomedical Robotics and Biomechatronics (Biorob), pages 414-419, Enschede, The Netherlands.

Damsgaard, M., Rasmussen, J., Christensen, S. T., Surma, E., and de Zee, M. (2006). Analysis of musculoskeletal systems in the AnyBody modeling system. Simulation Modelling Practice and Theory, 14(8):1100-1111.

de Sousa, A. C. C., Freire, J. P. C. D., and Bo, A. P. L. (2019). Integrating hip exosuit and FES for lower limb rehabilitation in a simulation environment. IFAC-PapersOnLine, 51(34):302-307.

de Sousa, A. C. C., Ramos, F. M., Dorado, M. C. N., Fonseca, L. O., and Bó, A. P. L. (2016). A comparative study on control strategies for fes cycling using a detailed musculoskeletal model. IFAC-PapersOnline, pages 204-209.

Delp, S., Loan, J., Hoy, M., Zajac, F., Topp, E., and Rosen, J. (1990). An interactive graphics-based model of the lower extremity to study orthopaedic surgical procedures. IEEE Transactions on Biomedical Engineering, 37(8):757-767.

Delp, S. L., Anderson, F. C., Arnold, A. S., Loan, P., Habib, A., John, C. T., Guendelman, E., and Thelen, D. G. (2007). Opensim: Open-source software to create and analyze dynamic simulations of movement. IEEE Transactions on Biomedical Engineering, 54(11):1940-1950.

Diaz, I., Gil, J. J., and Sanchez, E. (2011). Lower-limb robotic rehabilitation: Literature review and challenges. Journal of Robotics, 2011.

dos Santos, W. M., Caurin, G. A. P., and Siqueira, A. A. G. (2017a). Design and control of an active knee orthosis driven by a rotary series elastic actuator. Control Engineering Practice, 58:307-318.

dos Santos, W. M., Nogueira, S. L., de Oliveira, G. C., Pena, G. G., and Siqueira, A. A. G. (2017b). Design and evaluation of a modular lower limb exoskeleton for rehabilitation. In 2017 International Conference on Rehabilitation Robotics (ICORR). IEEE.

Frey, S. H., Fogassi, L., Grafton, S., Picard, N., Rothwell, J. C., Schweighofer, N., Corbetta, M., and Fitzpatrick, S. M. (2011). Neurological principles and rehabilitation of action disorders. Neurorehabilitation and Neural Repair, 25(5):6-20.

Guzmán, C. H., Blanco, A., Brizuela, J. A., and Gómez, F. A. (2017). Robust control of a hip-joint rehabilitation robot. Biomedical Signal Processing and Control, 35:100-109. 
Handford, M. L. and Srinivasan, M. (2018). Energy-optimal human walking with feedback-controlled robotic prostheses: A computational study. IEEE Transactions on Neural Systems and Rehabilitation Engineering, 26(9):1773-1782.

Jutinico, A. L., Escalante, F. M., Jaimes, J. C., Terra, M. H., and Siqueira, A. A. G. (2018). Markovian robust compliance control based on electromyographic signals. In 2018 7th IEEE International Conference on Biomedical Robotics and Biomechatronics (Biorob), Enschede, The Netherlands.

Jutinico, A. L., Jaimes, J. C., Escalante, F. M., Perez-Ibarra, J. C., Terra, M. H., and Siqueira, A. A. G. (2017). Impedance control for robotic rehabilitation: A robust markovian approach. Frontiers in Neurorobotics, 11:43.

Kainz, H., Modenese, L., Lloyd, D., Maine, S., Walsh, H., and Carty, C. (2016). Joint kinematic calculation based on clinical direct kinematic versus inverse kinematic gait models. Journal of Biomechanics, 49(9):1658-1669.

Khamar, M. and Edrisi, M. (2018). Designing a backstepping sliding mode controller for an assistant human knee exoskeleton based on nonlinear disturbance observer. Mechatronics, 54:121-132.

Kim, J. and Kim, J. (2018). An impedance control of human ankle joint using functional electrical stimulation. In 2018 7th IEEE International Conference on Biomedical Robotics and Biomechatronics (Biorob), pages 1230-1235, Enschede, The Netherlands.

Mackay, J. and Mensah, G. A. (2004). The Atlas of Heart Disease and Stroke. World Health Organization.

Mansouri, M. and Reinbolt, J. A. (2012). A platform for dynamic simulation and control of movement based on opensim and matlab. Journal of biomechanics, 45:1517-21.

Michaud, B. and Begon, M. (2021). biorbd: A c++, python and MATLAB library to analyze and simulate the human body biomechanics. Journal of Open Source Software, 6(57):2562.

MS (2018). Ministério da saúde - panorama do avc no brasil.

Nunes, P. F., dos Santos, W. M., and Siqueira, A. A. G. (2018). Control strategy based on kinetic motor primitives for lower limbs exoskeletons. In 10th IFAC Symposium on Biological and Medical Systems. International Federation of Automatic Control.

NUNES, P. F., SANTOS, W. M., NOGUEIRA, S., and SIQUEIRA, A. A. G. (2017). Analyzing motor primitives of healthy subjects wearing a lower limb exoskeleton. In International Congress of Mechanical Engineering - COBEM, volume 1, Curitiba.

Pastore, A., Pierella, C., Artoni, F., Pirondini, E., Coscia, M., Casadio, M., and Micera, S. (2018). Motor intention decoding during active and robot-assisted reaching. In 2018 7th IEEE International Conference on Biomedical Robotics and Biomechatronics (Biorob), pages 312-317, Enschede, The Netherlands.

Peña, G. G. (2017). Controle de Impedância Adaptativo Dirigido por EMG para Reabilitação Robótica. phdthesis, Universidade de São Paulo, São Carlos. 
Peña, G. G., Consoni, L. J., dos Santos, W. M., and Siqueira, A. A. G. (2019). Feasibility of an optimal emg-driven adaptive impedance control applied to an active knee orthosis. Robotics and Autonomous Systems, (112):98-108.

Ramos-Lima, M., Brasileiro, I., Lima, T., and Braga-Neto, P. (2018). Quality of life after stroke: impact of clinical and sociodemographic factors. Clinics, 73.

Rangel, E. S. S., Belasco, A. G. S., and Diccini, S. (2013). Quality of life of patients with stroke rehabilitation. Acta Paulista de Enfermagem, 26(2):205-212.

SAFANELli, J., VIEIRA, L. G. D. R., de ARAUJO, T., MANCHOPE, L. F. S., KUHLHOFF, M. H. R., NAGEL, V., CONFORTO, A. B., SILVA, G. S., MAZIN, S., de MAGALHÃES, P. S. C., and CABRAL, N. L. (2019). The cost of stroke in a public hospital in brazil: a one-year prospective study. Arquivos de Neuro-Psiquiatria, 77(6):404-411.

Seth, A., Hicks, J. L., Uchida, T. K., Habib, A., Dembia, C. L., Dunne, J. J., Ong, C. F., DeMers, M. S., Rajagopal, A., Millard, M., Hamner, S. R., Arnold, E. M., Yong, J. R., Lakshmikanth, S. K., Sherman, M. A., Ku, J. P., and Delp, S. L. (2018). OpenSim: Simulating musculoskeletal dynamics and neuromuscular control to study human and animal movement. PLOS Computational Biology, 14(7):e1006223.

Trinler, U., Schwameder, H., Baker, R., and Alexander, N. (2019). Muscle force estimation in clinical gait analysis using AnyBody and OpenSim. Journal of Biomechanics, 86:55-63.

WHO (2011). World health organization - world report on disability.

WHO (2018). World health organization - the top 10 causes of death.

Wolpert, D. M., Miall, R. C., and Kawato, M. (1998). Internal models in the cerebellum. Trends in Cognitive Sciences, 2(9):338-347. 